\title{
Epigenetic signatures of somatic cell nuclear transfer-derived embryonic stem cells
}

\author{
EUI-MAN JUNG ${ }^{1}$, HYE YOUNG SON ${ }^{2}$, MIN KWON JEUNG ${ }^{1,2}$, CHANG KYU LEE ${ }^{3}$, \\ SANG HWAN HYUN ${ }^{1}$ and EUI-BAE JEUNG ${ }^{1}$
}

\author{
${ }^{1}$ College of Veterinary Medicine, Chungbuk National University, Cheongju, Chungbuk 361-763; ${ }^{2}$ Sooam Biotech Research \\ Foundation, Wonsam-myeon, Cheoin-gu, Yongin-si, Gyeonggi-do 449-872; ${ }^{3}$ Department of Agricultural \\ Biotechnology, Seoul National University, Gwanak-gu, Seoul 151-921, Republic of Korea
}

Received June 22, 2011; Accepted July 28, 2011

DOI: $10.3892 /$ ijmm.2011.772

\begin{abstract}
Some human embryonic stem cell lines have shown genomic instabilities over long-term culture. To study the controversial origin of the SCNT-hES-1 line, which was derived from autologous somatic cell nuclear transfer (SCNT), we compared the expression and methylation patterns of imprinted genes in the SCNT-hES-1 cells with the donor's somatic cells by semi-quantitative RT-PCR, real-time PCR and bisulfite sequencing. Examined imprinted genes were H19, GNAS, $S L C 22 A 18, U B E 3 A$ and ZNF264 for maternally expressed genes, and IGF2, SNRPN, PEG3, PEG10, MEST, MAGEL2 and $A R H I$ for paternally expressed genes, respectively. We found that the expression of imprinted genes in the SCNT-hES-1 cell line is comparable to that in the donor's somatic cells, and that its methylation patterns are similar to those of other SCNT-products. Therefore, the present study indicates that the SCNT-hES-1 line was derived from SCNT.
\end{abstract}

\section{Introduction}

The somatic cell nuclear transfer (SCNT) technique has been widely acknowledged for its potential utility for regenerative medicine (1-5). Hwang et al (4) claimed that they produced the first human embryonic stem cell line, SCNT-hES-1, via autologous SCNT, by transferring the donor's own somatic cell into the donor's own enucleated oocyte. Because autologous SCNT provides the molecular and genetic identity for a reconstructed SCNT embryo, it may decrease deficient epigenetic reprogramming of the donor cell nucleus in an enucleated oocyte. Also, to overcome inefficiencies of human SCNT, autologous SCNT was performed in the production of SCNT-hES-1 (4).

Correspondence to: Dr Eui-Bae Jeung, Laboratory of Veterinary Biochemistry and Molecular Biology, College of Veterinary Medicine, Chungbuk National University, Cheongju, Chungbuk 361-763, Republic of Korea

E-mail: ebjeung@chungbuk.ac.kr

Key words: epigenetic, imprinted gene, somatic cell nuclear transfer, methylation, SCNT-hES-1
However, for this reason, there have been disputes over its origin.

The Seoul National University Investigation Committee (SNUIC) has insisted that parthenogenetic SCNT-hES-1 were generated by the re-influx of the first polar body using only an analysis of short tandem repeat (STR) DNA. However, Kim et al (6) claimed that there was no scientific evidence for SNUIC's assumption stated above, the re-influx of the first polar body, and that SCNT-hES-1 was created by parthenogenesis derived from inhibition of second polar body's extrusion as supported by analysis of single nucleotide polymorphisms (SNP) and methylation patterns in the cell line. Their SNP results indicated that the line appeared to be homozygous in the centromeric region of nearly every chromosome (6). Therefore, the parthenogenic origin of SCNT-hES-1 is unclear. They also insisted that the SCNT-hES-1 line originated from parthenogenesis based on an analysis of DNA methylation patterns, despite the absence of comparison with the donor's somatic cells or of the IVF-ES line as a control (6). Indeed, it is difficult to determine the origin of SCNT products (cloned embryos or ES cells) by methylation patterns alone, because there are aberrant methylation patterns in various genomic regions after SCNT (7). It has also been questioned why they used the SCNT-hES-1 line with an abnormal karyotype $(45, \mathrm{XO})$ for their experiments (6), despite the existence of the line with normal karyotype (46, XX).

To disclose the nature of SCNT-hES-1, we used genomic imprinting and methylation patterns to compare the SCNT-hES-1 passage-70 (70P, normal karyotype), SCNT-hES-1 passage-140 (140P, abnormal karyotype) lines and the donor's somatic cells.

\section{Materials and methods}

Cell culture. Three lines of hES cells, SCNT-hES-1 at passage 70 (70P), SCNT-hES-1 at passage 140 (140P) and H9 (WiCell, Wisconsin, USA) were cultured in hES medium DMEM/F12 (Invitrogen, CA, USA) medium supplemented with $20 \%$ knockout serum replacement (KSR; Invitrogen), $2 \mathrm{mM}$ L-glutamine (Invitrogen), $0.1 \mathrm{mM} \beta$-mercaptoethanol (Invitrogen), 1\% MEM non-essential amino acids (Invitrogen), $1 \%$ antibioticantimycotic (Invitrogen) containing cytokines, $10 \mathrm{ng} / \mu \mathrm{l}$ human recombinant bFGF (hrbFGF; Invitrogen) on a mitomycin-C 


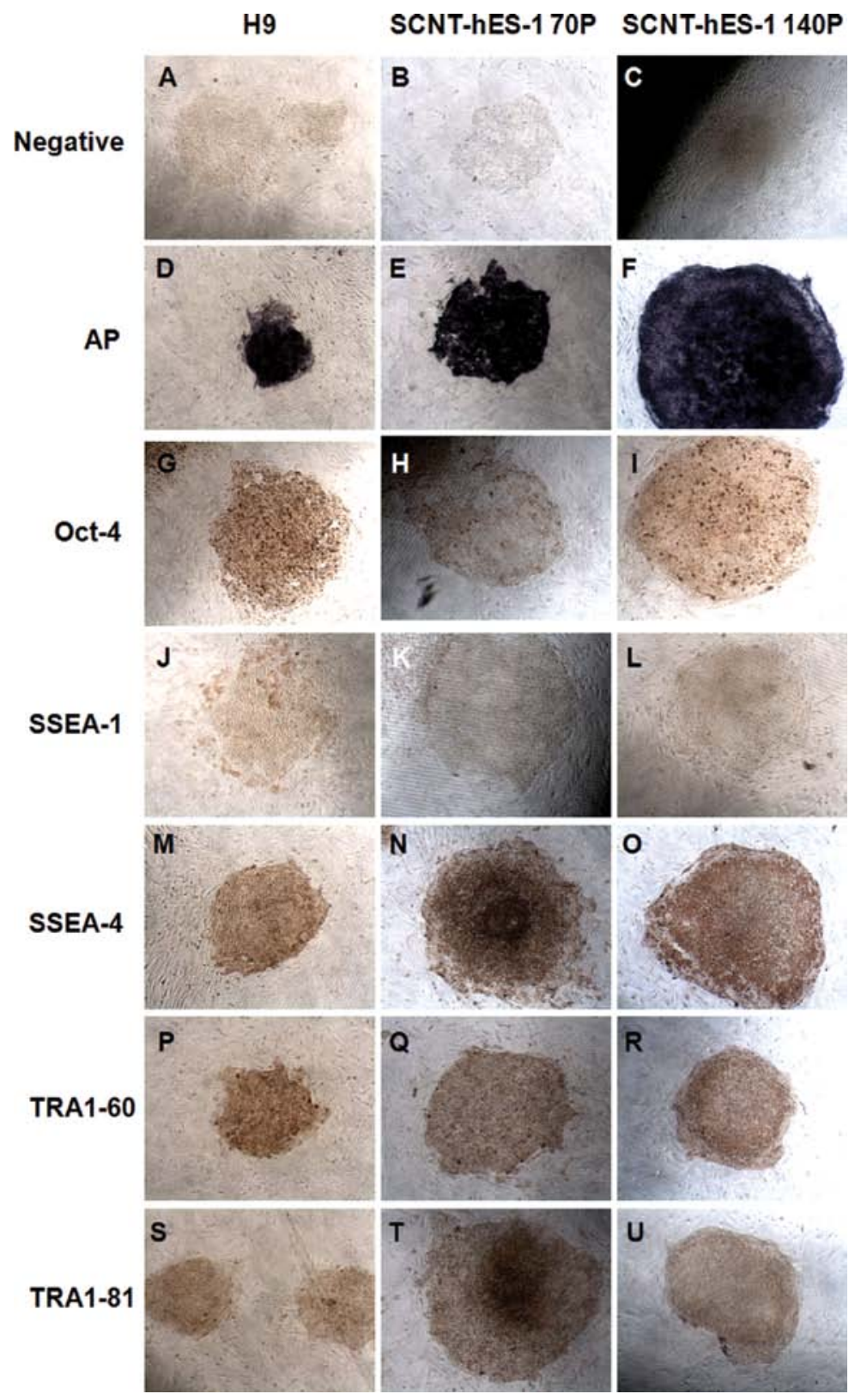

Figure 1. Expression of pluripotent stem cell markers in SCNT-hES-1 ES cells at 79 (70P) and 131 (140P) passages. SCNT-hES-1 cells expressed all analyzed cell surface markers, including (E and F) AP (alkaline phosphatase), (H and I) Oct-4, (N and O) SSEA-4, (Q and R) TRA1-60 and (T and U) TRA1-81, but not (K and L) SSEA-1. The IVF-derived hES cells (H9) were used for comparison and also positively expressed (D) AP, (G) Oct-4, (M) SSEA-4, (P) TRA1-60 and (S) TRA1-81 but not (J) SSEA-1. Negative controls not treated with the first antibodies are shown (A, B and C) (x40).

(Roche, Mannheim, Germany) inactivated murine embryonic fibroblast (MEF) feeder layer. The donor's somatic cells were cultured in DMEM (Invitrogen) medium with $10 \%$ fetal bovine serum (FBS; Invitrogen), $0.1 \mathrm{mM} \beta$-mercaptoethanol and $1 \%$ antibiotic-antimycotic. A donor fibroblast cell was prepared as a control in all experiments. The SCNT-hES-1 lines displayed stem cell morphology and expressed ES cell pluripotent markers, including AP, Nanog, Oct-4, SSEA-4, TRA1-60 and TRA1-81, but not a negative cell surface marker, SSEA-1 (Fig. 1).

STR analysis. To examine the genomic identity of the SCNT-hES-1 lines, we analyzed 15 STR markers, and the STR result demonstrated that DNAs from SCNT-hES-1 lines matched with the donor's DNA (Table I). The genomic DNA samples were isolated from cell lines using a Qiagen Genomic
DNA kit (Qiagen, Hilden, Germany) and submitted to the HumanPass Co. (Seoul, Korea) for STR analysis of fifteen STR loci. An STR typing process was performed according to the manufacturer's protocols.

Karyotyping analysis. ES colonies were cultured in hES medium on a 4-well plate without feeder layers. After 2 days, the cells were incubated with $15 \mu \mathrm{l}$ of colcemid (KaryoMax ${ }^{\circledR}$ Colcemid ${ }^{\circledR}$ Solution, Invitrogen) for $24 \mathrm{~h}$ in a humidified environment of $5 \% \mathrm{CO}_{2}$ in air at $37^{\circ} \mathrm{C}$. The cells were washed with PBS and trypsinized for single-cell separation, and then the pellets were treated with hypotonic solution (1\% sodium citrate, Sigma) for $30 \mathrm{~min}$ at room temperature. The cells were fixed with methanol-acetic acid (1:1). Then, the cell suspension was put onto a glass slide and broken by the drop of methanol-acetic 
Table I. STR analysis of the SCNT-hES-1 and its somatic cell donor.

\begin{tabular}{|c|c|c|c|c|c|c|}
\hline \multirow{2}{*}{$\frac{\text { STR Loci }}{\text { D8S1179 }}$} & \multicolumn{2}{|c|}{ Donor } & \multicolumn{2}{|c|}{ SCNT-hES-1 70P } & \multicolumn{2}{|c|}{ SCNT-hES-1 140P } \\
\hline & 10 & 11 & 10 & 11 & 10 & 11 \\
\hline $\mathrm{D} 21 \mathrm{~S} 11^{\mathrm{a}}$ & 28 & 32.2 & 32.2 & 32.2 & 32.2 & 32.2 \\
\hline D7S820 & 8 & 11 & 8 & 11 & 8 & 8 \\
\hline CSF1PO & 12 & 13 & 12 & 13 & 12 & 13 \\
\hline D3S1358 & 16 & 18 & 16 & 18 & 16 & 18 \\
\hline TH01 & 6 & 9 & 6 & 9 & 6 & 9 \\
\hline D13S317 & 8 & 9 & 8 & 9 & 8 & 9 \\
\hline D16S539 & 9 & 12 & 9 & 12 & 9 & 12 \\
\hline D2S1338 & 18 & 19 & 18 & 19 & 18 & 19 \\
\hline D19S433 ${ }^{a}$ & 13 & 13.2 & 13 & 13 & 13 & 13 \\
\hline $\mathrm{vWA}^{\mathrm{a}}$ & 16 & 17 & 17 & 17 & 17 & 17 \\
\hline TPOX & 8 & 8 & 8 & 8 & 8 & 8 \\
\hline D18S51 & 15 & 16 & 15 & 16 & 15 & 16 \\
\hline D5S818 & 10 & 11 & 10 & 11 & 10 & 11 \\
\hline FGA & 21 & 23 & 21 & 23 & 21 & 23 \\
\hline
\end{tabular}

${ }^{\text {aSCNT}-h E S-1 ~ a t ~ p a s s a g e ~} 70(70 \mathrm{P})$ and passage $140(140 \mathrm{P})$ were homozygous at these loci; ${ }^{\mathrm{b} S C N T}-\mathrm{hES}-1$ 140P was homozygous at this locus .

Table II. Primers for semi-quantitative RT-PCR.

Primer sequences

\begin{tabular}{lll}
\cline { 2 - 3 } Genes & \multicolumn{1}{c}{ Forward } & Reverse \\
\hline H19 & ttacaaccactgcactacctgact & gctcagaaacaaagagacagaagg \\
GNAS & ctcgccataattacaacgacct & ctcttcctcgtactctaggcactc \\
MEG3 & cttgaaaggcctgtctacacttg & caaaccaggaaggagacgagag \\
SLC22A18 & atgtccagcgtcttccacttct & gacaagggtattgatagcaacctg \\
UBE3A & ggagtatgaagggaatgtggaa & ccatatgaaaacctctccgaaa \\
ZNF264 & gattcatagtggagagaagccta & agaagttctcgaagggtaactgag \\
IGF2 & ctcctggagacgtactgtgcta & gtcttgggtgggtagagcaatc \\
SNRPN & gagaacttggtatccatgactgtg & ctcttccctgtggagtcattacct \\
PEG3 & agctttgaaatggacagagaggac & ggtaggcacttctcttggatctt \\
PEG10 & gtgcattcacattgagagaagg & gtagtgacctcctgttccacagt \\
MEST & gacgggaacttagtcattgacag & tctagctgtggatagtggctaatg \\
MAGEL2 & gtgtcagggaggatctgatcttta & gctctgtggatctttcttatggag \\
IPW & gtggatagatgcacacaaacacac & tatagggaggttcattgcacag \\
ARHI & ctcagtcaccaagaaggaaacc & gtaattcagcagcatgtggaac \\
GAPDH & ggtgtgaaccatgagaagtatgac & agtagaggcagggatgatgttct
\end{tabular}

acid. The slides with chromosome spreads were stained in $20 \%$ Giemsa staining solution (Sigma) for $5 \mathrm{~min}$, rinsed, and observed under the microscope at a x1,000 magnification with oil immersion.

Semi-quantitative $R T-P C R$ and real-time $P C R$ using TaqMan $^{\mathrm{TM}}$. Total-RNA was prepared from the donor's somatic cells, SCNT-hES-1 70P, SCNT-hES-1 140P and H9 cells using the TRIzol reagent. A first-strand complementary DNA
(cDNA) library was prepared by subjecting total-RNA (1 ng) to reverse transcription using MMLV reverse transcriptase (Invitrogen Life Technologies, Inc.) and random primers (9-mer, Takara Bio, Inc., Shiga, Japan). To determine the conditions for the logarithmic phase of PCR amplification for target mRNA, aliquots $(1 \mu \mathrm{g})$ were amplified using different numbers of cycles. The GAPDH gene was PCR-amplified to rule out the possibility of RNA degradation and was used as a control for variation in mRNA concentrations in the RT 
Table III. Assay on-Demand ${ }^{\mathrm{TM}}$ probes for real-time PCR.

\begin{tabular}{lc}
\hline Genes & Assay ID \\
\hline H19 & Hs00262142_g1 \\
GNAS & Hs00255603_m1 \\
MEG3 & Hs00292028_m1 \\
SLC22A18 & Hs00180039_m1 \\
UBE3A & Hs00166580_m1 \\
ZNF264 & Hs00185667_m1 \\
IGF2 & Hs00171254_m1 \\
IGF2R & Hs00181419_m1 \\
SNRPN & Hs00256090_m1 \\
PEG3 & Hs00300418_s1 \\
PEG10 & Hs00248288_s1 \\
MEST & Hs00853380_g1 \\
MAGEL2 & Hs00255922_s1 \\
IPW & Hs01374548_g1 \\
ARHI & Hs00190723_m1 \\
GAPDH & Hs00266705_g1 \\
\hline
\end{tabular}

reaction. A linear relationship between PCR products and amplification cycles was observed for the target mRNAs. GAPDH and the target genes were quantified using 28 and 30 cycles, respectively. The cDNA was amplified in a $20 \mu \mathrm{l}$ PCR reaction containing 1 unit Taq polymerase (iNtRON Bio, Inc.), $2 \mathrm{mM}$ dNTP and 10 pmol specific primers. PCR reactions were denatured at $95^{\circ} \mathrm{C}$ for $30 \mathrm{sec}$, annealed at 60 or $62^{\circ} \mathrm{C}$ for $30 \mathrm{sec}$ and extended at $72^{\circ} \mathrm{C}$ for $30 \mathrm{sec}$. The oligonucleotide sequences of specific primers are shown in Table II. PCR products $(8 \mu \mathrm{l})$ were fractionated on a $2 \%$ agarose gel, stained with ethidium bromide and photographed under UV illumination. The photograph was scanned using Gel Doc EQ (Bio-Rad Laboratories, Inc.). A real-time PCR was performed in a $20 \mu \mathrm{l}$ reaction volume containing $10 \mu \mathrm{l}$ of TaqMan Universal PCR Master mix (Applied Biosystems, Foster, CA, USA), $1 \mu 1$ of 20X Assays-on-Demand ${ }^{\text {TM }}$ Gene Expression assay mix (Applied Biosystems) and $1 \mu \mathrm{l}$ cDNAs (Table III). Amplification was carried out using a 7300 Real-Time PCR System (Applied Biosystems) and the following cycle parameters: initial denaturation at $50^{\circ} \mathrm{C}$ for $2 \mathrm{~min}, 90^{\circ} \mathrm{C}$ for $10 \mathrm{~min}$, 40 cycles of denaturation at $95^{\circ} \mathrm{C}$ for $15 \mathrm{sec}$, and annealing and extension at $60^{\circ} \mathrm{C}$ for $1 \mathrm{~min}$. The expression levels were determined using RQ software (Applied Biosystems). The expression levels of amplified genes were normalized to that of GAPDH.

Sodium bisulfite modification and bisulfite sequencing. Genomic DNAs from the donor's somatic cells, SCNT-hES-1 70P, SCNT-hES-1 140P and H9 cells were isolated using a G-DEX ${ }^{\mathrm{TM}}$ IIc Genomic DNA Extraction kit (Intron Biotechnology, Suwon, Korea). Two micrograms of DNA was modified by sodium bisulfite using an EZ DNA MethylationGold $^{\mathrm{TM}}$ kit (Zymo Research, Irvine, CA) according to the manufacturer's instructions. To perform bisulfite sequencing analysis in order to confirm the methylation of $\mathrm{CpG}$ sites of
Table IV. Primers for bisulfite sequencing.

\begin{tabular}{ll}
\hline Genes & \multicolumn{1}{c}{ Primer sequences } \\
\hline H19 & $\begin{array}{l}\text { Forward: gtattgtgggaggggttagtatag } \\
\text { Reverse: actttattctctaatccaaaatccttatat }\end{array}$ \\
SLC22A18 & Forward: tggttgaggtattattttttgaga \\
& Reverse: ataaaaccctaacaccctaaactt \\
FNRPN & Forward: tttttaatttgatagagagttttgg \\
& Reverse: acaacaaacaaactcaaaactaatacac \\
MEST & Forward: ttttttgggaatagggtgaag \\
& Reverse: tttccaacctccaaaactaactatc \\
\hline
\end{tabular}

bisulfite-modified DNA, the DNA was amplified in a $20 \mu 1$ reaction with the following primer sets, the design of which was based on the location of the probe position for genomewide methylation analysis and the MethPrimer program (http:// www.urogene.org/methprimer/index.html). The primers are listed in Table IV. The reactions were carried out for 33 cycles. The cycling parameters were as follows: denaturation at $95^{\circ} \mathrm{C}$ for $45 \mathrm{sec}$, annealing at $58^{\circ} \mathrm{C}$ for $45 \mathrm{sec}$, and extension at $72^{\circ} \mathrm{C}$ for $45 \mathrm{sec}$. The PCR products were cloned into the yT\&A Cloning Vector (RBC Real-Biotech Corp., Taipei, Taiwan), and ten clones were randomly chosen for direct sequencing.

Statistics. Data were analyzed by non-parametric one-way analysis of variance using the Kruskal-Wallis test, followed by the Dunnett's test for multiple comparisons to vehicle. All statistical analyses were performed with SPSS for Windows Edition (SPSS, Chicago, IL). A P-value $<0.05$ was considered statistically significant.

\section{Results}

Genomic identity of the SCNT-hES-1 line by STR analysis. The genomic identity of the SCNT-hES-1 line, as analyzed by 15 STR markers, verified that the DNA from the SCNT-hES-1 line matched that from the donor (Table I). However, three makers (D21S11, D19S433, vWA) appeared to show homozygosity in SCNT-hES-1 70P. There were four homozygosity-markers, including an additional maker (D7S820), in SCNT-hES-1 140P. A similar result was obtained in the initial investigations of SNUIC, in which STR marker analysis of 40 markers showed that 8 markers had been converted from heterozygosity to homozygosity. A conventional cytogenetic G-banding karyotyping was performed on SCNT-hES-1 70P and 140P. The karyotype of SCNT-hES-1 70P was consistent with a 46, XX karyotype; however, an abnormal karyotype (45, XO; X chromosome deletion) was observed in SCNT-hES-1 140P (Fig. 2). These results indicate that the SCNT-hES-1 line may have developed genomic instability over long-term culture.

Expression pattern of paternally imprinted genes in the SCNT-hES-1 cell line. We performed real-time PCR and semiquantitative RT-PCR to investigate whether imprinted genes were expressed in the donor line, the SCNT-hES-1 70P, the SCNT-hES-1 140P and H9 cells. Real-time PCR analysis showed 
A Donor 10P

B SCNT-hES-170P

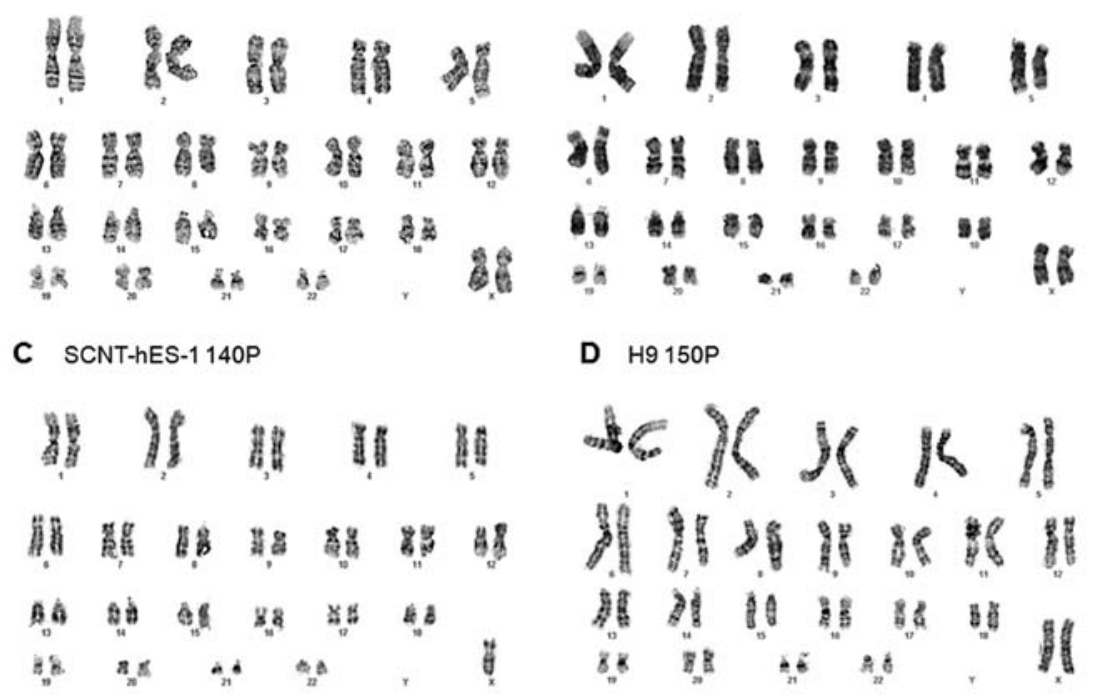

Figure 2. The G-banded karyotyping of the SCNT-hES-1, donor, and H9 cell lines. (A, B and D) A donor somatic cell line at passage 10 (10P), SCNT-1 at passage 70 (70P) and $\mathrm{H} 9$ at passage 150 (150P) all have a normal 46, XX karyotype. (C) The SCNT-hES-1 line at passage 140 (140P) has a 45, XO karyotype.

\section{A Maternally expressed imprinted genes}
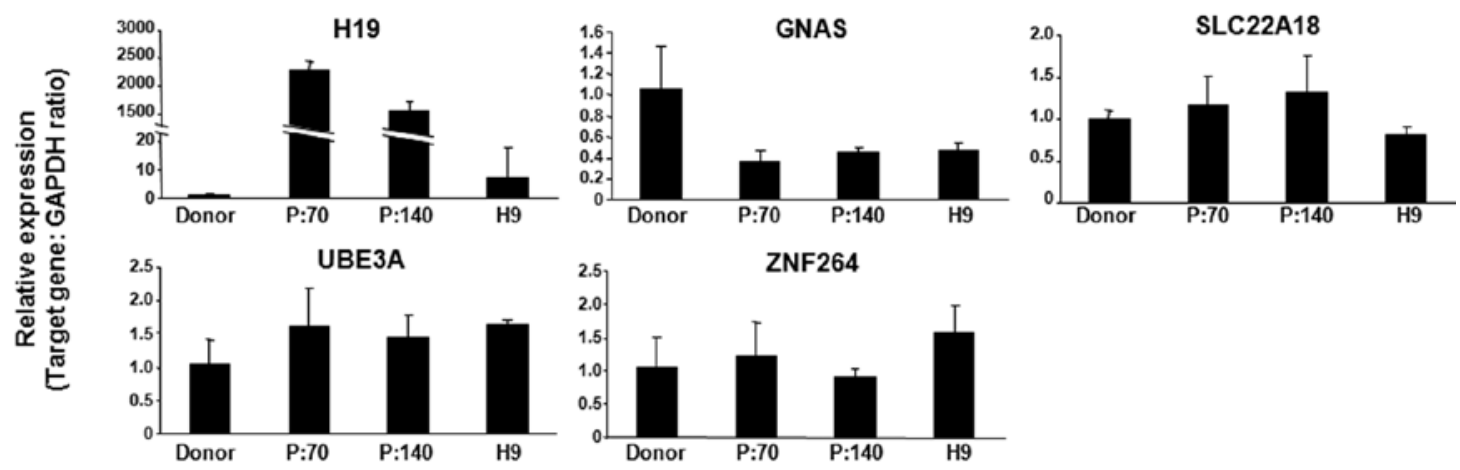

B Paternally expressed imprinted genes
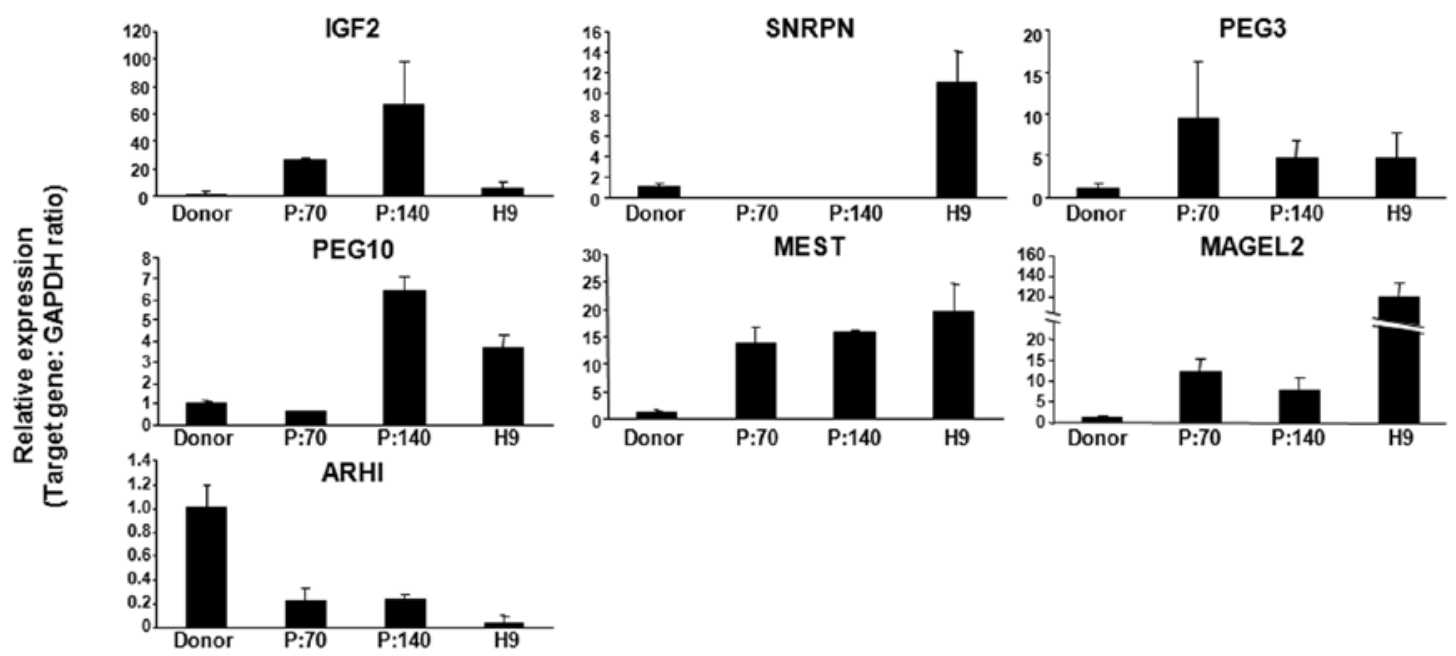

Figure 3. Expression of imprinted genes in the donor's somatic cells (Donor), SCNT-hES-1 70P (70P), SCNT-hES-1 140P (140P) and the IVF (H9) cell line as measured by real-time PCR. Amplification of maternally expressed (H19, GNAS, SLC22A18, ZNF264 and UBE3A) (A) and paternally expressed (IGF2, SNRPN, PEG3, PEG10, MEST, MAGEL2 and ARHI) genes (B). Data represent the mean \pm SEM of triplicate measurements of all samples and are presented as a percentage of the expression of an internal control, GAPDH. 


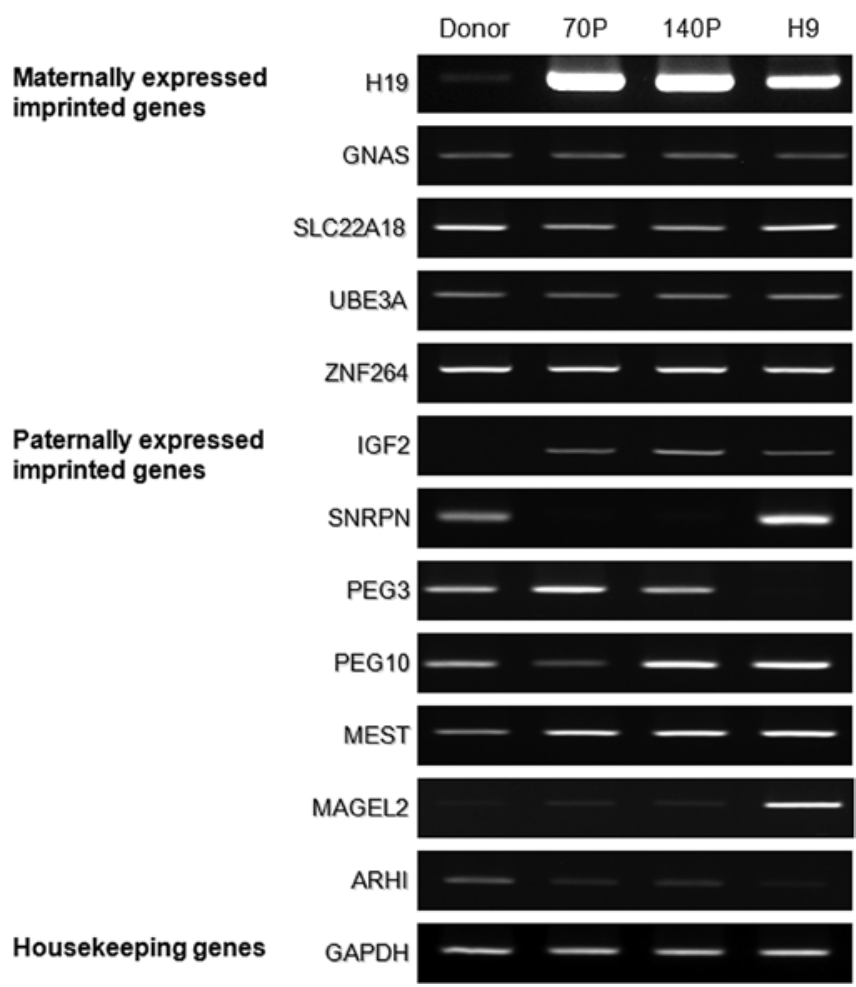

Figure 4. Expression of imprinted genes in the donor's somatic cells (Donor), SCNT-hES-1 70P (70P), SCNT-hES-1 140P (140P) and the IVF (H9) line by RT-PCR. Amplification of (A) maternally expressed (H19, GNAS, SLC22A18, ZNF264 and UBE3A) and (B) paternally expressed (IGF2, SNRPN, PEG3, PEG10, MEST, MAGEL2 and ARHI) genes.

that all three cell types expressed the maternal genes (Fig. 3A). The expression levels of all analyzed maternally expressed genes in the SCNT-hES-1 lines at 70P and 140P were similar to those in the donor and $\mathrm{H} 9$ lines as would be expected, except H19. Six of the seven paternally expressed genes, including $I G F 2$, PEG3, PEG10, MEST, MAGEL2 and ARH1, were normally expressed in both SCNT-hES-1 lines; the exception was SNRPN (Fig. 3B). A semi-quantitative PCR confirmed the presence of maternal and paternal gene transcripts, as shown in Fig. 4.

Aberrant methylation patterns in various genes of the SCNT-hES1 line. We have performed a genome-wide analysis of the methylation levels of $\mathrm{CpG}$ dinucleotides in the SCNT-hES-1, donor and H9 cell lines using a bisulfite sequencing analysis. The methylation levels of the maternally expressed $H 19$ gene were identical among the SCNT-hES-1 lines and appeared to be lower than the levels observed in the donor cells and $\mathrm{H} 9$ controls. However, methylation levels of the maternally expressed SLC22A18 gene were identical among the SCNT-hES-1 lines as well as the donor and H9 controls (Fig. 5A). The methylation levels of the paternally expressed SNRPN and MEST in the SCNT-hES-1 lines showed methylation levels opposite to the donor cells, but appeared to be similar to those in the H9 line. (Fig. 5B)

\section{Discussion}

This study demonstrated that the SCNT-hES-1 cell lines showed genomic instability over long-term culture, and six of the seven paternally expressed genes, including $I G F 2, P E G 3$, PEG10, MEST, MAGEL2 and ARH1, were normally expressed in both SCNT-hES-1 lines.

Recently, a considerable number of studies has reported similar abnormal karyotypes, with $\mathrm{Y}$ chromosome deletion observed in SCNT monkey ES cell lines (8), and recurrent genomic instability has been reported in hES cell lines over long-term culture (9). Genomic imprinting is a genetic phenomenon by which certain genes are expressed in a parent-of-origin-specific manner. Imprinted genes are either expressed only from the allele inherited from the mother, or in other instances from the allele inherited from the father. Imprinting is an important genetic mechanism in mammals (10). Unlike in vivo-fertilized products, however, SCNTproducts demonstrate an abnormal expression and methylation patterns of imprinted genes.

Two groups have reported the isolation of hES cell lines (hPES) derived from parthenogenesis $(11,12)$. The expression patterns of imprinted genes in these hPES lines were analyzed to confirm their derivation from parthenogenesis. In these studies, the paternally imprinted genes SNRPN and PEG1_2 were not detected in all hPES lines $(11,12)$. These results indicated that their cell lines were derived from a parthenogenetic ES line $(11,12)$, as indicated by the absence of paternally imprinted genes (13-15). Also, during the investigation and analysis of 59 human IVF-ES lines by the International Stem Cell Initiative (ISCI), of the investigated paternally expressed genes, IPW, SNRPN, KCNQ1OT1 and PEG3 showed monoallelic expression in all cells analyzed (16). Thus, we investigated whether imprinted genes (16) were expressed in the donor line, the SCNT-hES-1 70P, the SCNT-hES-1 140P and the $\mathrm{H} 9$ cells. Our results confirmed that maternal genes H19, GNAS, SLC22A18, UBE3A and ZNF264 mRNA were expressed in donor cells, SCNT-hES-1 70P, SCNT-hES-1 140P and $\mathrm{H} 9$ cells. $H 19$ mRNA expression was significantly higher in SCNT-hES-1 70P and 140P cells than in donor cells, and H9 cells also showed a higher expression than that of donor cells. These results suggested that the imprint stability of $H 19$ genes was also inherent to the individual cell line, rather than dependent on the cell culture condition (17-20). Also, our results confirmed that paternally expressed genes, including $I G F 2$, PEG3, PEG10, MEST, MAGEL2 and ARH1, were normally expressed in all lines. However, paternally expressed SNRPN mRNA was not expressed in either SCNT-hES-1 lines. RuggGunn et al (17) also suggested that some hES lines showed a loss of allele-specific expression, although the paternally imprinted genes $I P W, S N R P N$ and $K C N Q 1 O T 1$ were highly stable and thus appeared insensitive to perturbation. Thus, the expression pattern of paternally imprinted genes in the SCNT-hES-1 cell line indicates that it is not derived from parthenogenesis.

The levels of paternally expressed IGF2, PEG3 and $M A G E L 2$ in the SCNT-hES-1 lines appeared to be higher than the levels observed in the donor cells, but appeared to be similar to those in the H9 line. Similarly, two mouse studies observed aberrant DNA methylation patterns in SCNTproducts after long-term culture in vitro $(18,20)$. Unexpectedly, paternally expressed SNRPN was fully methylated in the SCNT-hES-1 lines, as compared to its normal methylation in the donor. It is presumed that the silencing of SNRPN in 
A DNA methylation status of maternally expressed imprinted genes
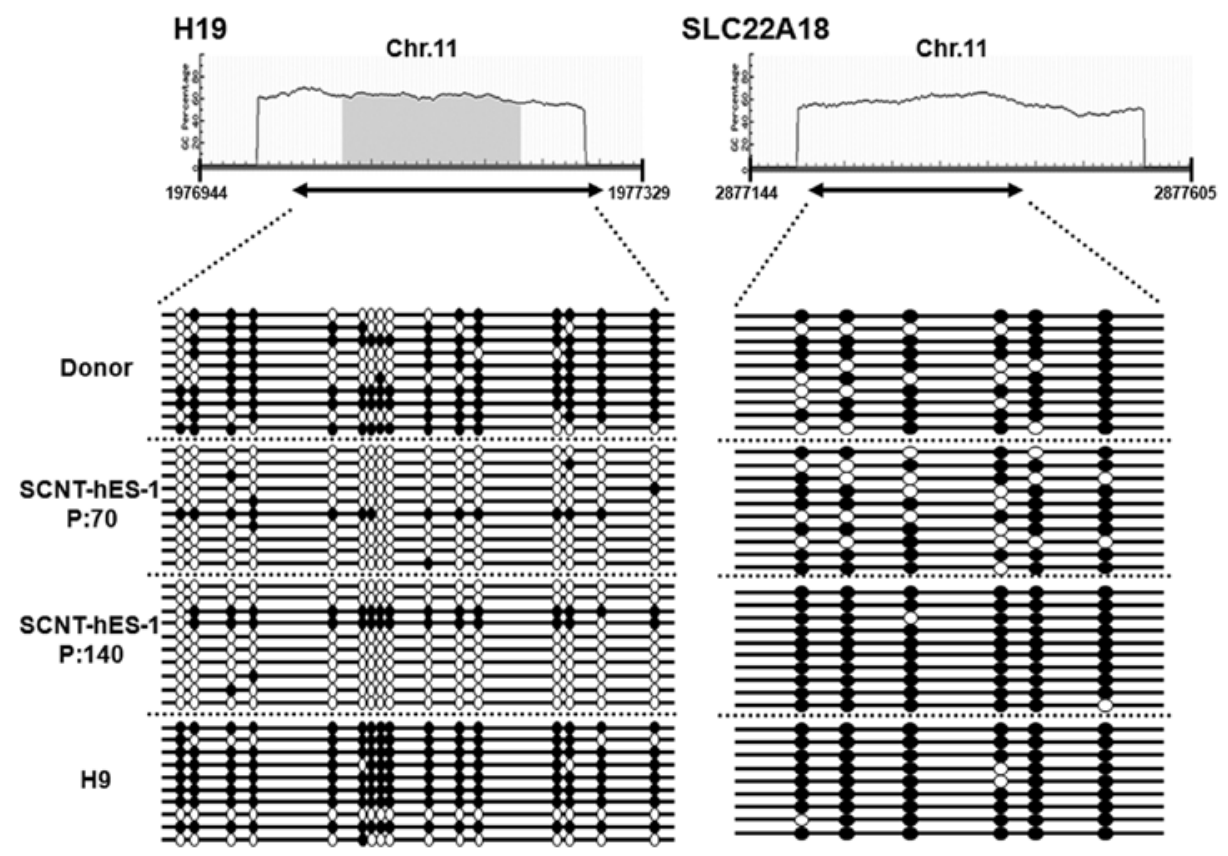

B DNA methylation status of paternally expressed imprinted genes
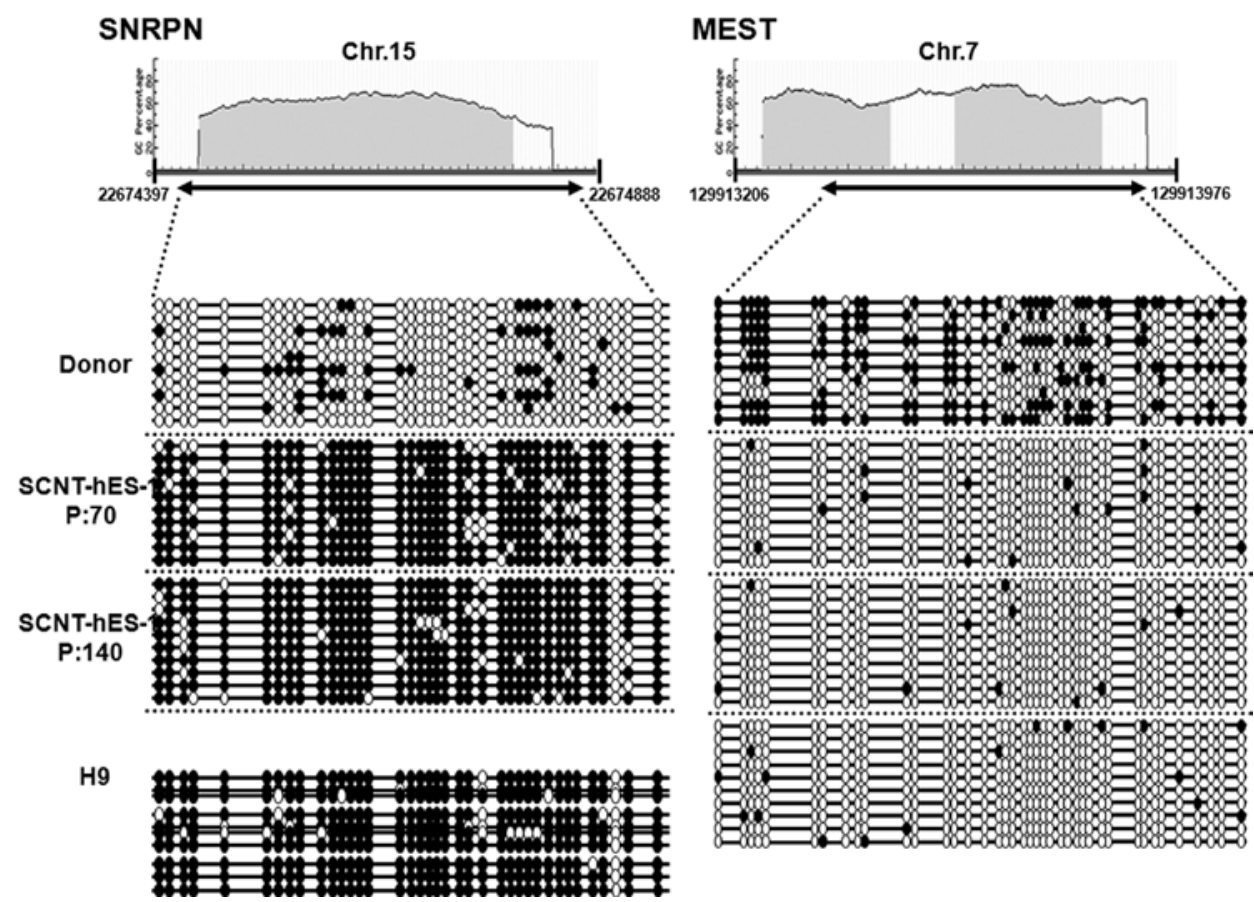

Figure 5. Methylation levels of several imprinted genes in the donor's somatic cells (Donor), SCNT-hES-1 70P (70P), SCNT-hES-1 140P (140P) and the IVF (H9) line by bisulfite sequencing analysis. The relative methylation levels of (A) the maternally expressed H19 and SLC22A18 gens and (B) the paternally expressed SNRPN and MEST genes were confirmed by a bisulfite sequencing analysis.

the SCNT-hES-1 line was possibly caused by the loss of one unmethylated allele of the $S N R P N$ gene, located on the region of chromosome 15q11.2. This evidence suggests that, unlike the parthenote, which has two maternal alleles, SCNT-hES-1 has both maternal and paternal alleles. Therefore, the aberrant methylation patterns in various genes of the SCNT-hES1 line are actually a side effect of the SCNT process.
In conclusion, our results demonstrate here that almost all paternally imprinted genes are expressed in the SCNT-hES-1 line and that this line's methylation patterns are similar to those of other SCNT-products. These results suggest that SCNT-hES-1 may possibly be a pluripotent hES cell line derived from an SCNT-cloned blastocyst higher than derivation from parthenogenesis. However, it would be desirable to 
establish the heterologous SCNT-hES line for the conclusive evidence.

\section{Acknowledgements}

The authors wish to thank Dr Mahendra S. Rao and Dr Soojung Shin for helpful advice.

\section{References}

1. Lanza RP, Cibelli JB and West MD: Human therapeutic cloning. Nat Med 5: 975-977, 1999.

2. Wakayama T, Tabar V, Rodriguez I, Perry AC, Studer L and Mombaerts P: Differentiation of embryonic stem cell lines generated from adult somatic cells by nuclear transfer. Science 292: 740-743, 2001.

3. Drukker M and Benvenisty N: The immunogenicity of human embryonic stem-derived cells. Trends Biotechnol 22: 136-141, 2004.

4. Hwang WS, Ryu YJ, Park JH, et al: Evidence of a pluripotent human embryonic stem cell line derived from a cloned blastocyst. Science 303: 1669-1674, 2004.

5. Byrne JA, Pedersen DA, Clepper LL, et al: Producing primate embryonic stem cells by somatic cell nuclear transfer. Nature 450 : 497-502, 2007.

6. Kim K, Ng K, Rugg-Gunn PJ, et al: Recombination signatures distinguish embryonic stem cells derived by parthenogenesis and somatic cell nuclear transfer. Cell Stem Cell 1: 346-352, 2007.

7. Kang YK, Koo DB, Park JS, et al: Aberrant methylation of donor genome in cloned bovine embryos. Nat Genet 28: 173-177, 2001.

8. Dighe V, Clepper L, Pedersen D, et al: Heterozygous embryonic stem cell lines derived from nonhuman primate parthenotes. Stem Cells 26: 756-766, 2008.
9. Spits C, Mateizel I, Geens M, et al: Recurrent chromosomal abnormalities in human embryonic stem cells. Nat Biotechnol 26: 1361-1363, 2008.

10. Reik W and Walter J: Genomic imprinting: parental influence on the genome. Nat Rev Genet 2: 21-32, 2001.

11. Mai Q, Yu Y,Li T, et al: Derivation of human embryonic stem cell lines from parthenogenetic blastocysts. Cell Res 17: 1008-1019, 2007.

12. Revazova ES, Turovets NA, Kochetkova OD, et al: Patient-specific stem cell lines derived from human parthenogenetic blastocysts. Cloning Stem Cells 9: 432-449, 2007.

13. Cibelli JB, Grant KA, Chapman KB, et al: Parthenogenetic stem cells in nonhuman primates. Science 295: 819, 2002.

14. Hernandez L, Kozlov S, Piras G and Stewart CL: Paternal and maternal genomes confer opposite effects on proliferation, cellcycle length, senescence, and tumor formation. Proc Natl Acad Sci USA 100: 13344-13349, 2003.

15. Vrana KE, Hipp JD, Goss AM, et al: Nonhuman primate parthenogenetic stem cells. Proc Natl Acad Sci USA 100 (Suppl 1): S11911-S11916, 2003.

16. Adewumi O, Aflatoonian B, Ahrlund-Richter L, et al: Characterization of human embryonic stem cell lines by the International Stem Cell Initiative. Nat Biotechnol 25: 803-816, 2007.

17. Rugg-Gunn PJ, Ferguson-Smith AC and Pedersen RA: Status of genomic imprinting in human embryonic stem cells as revealed by a large cohort of independently derived and maintained lines. Hum Mol Genet 16: R243-R251, 2007.

18. Chang G, Liu S, Wang F, et al: Differential methylation status of imprinted genes in nuclear transfer derived ES (NT-ES) cells. Genomics 93: 112-119, 2009.

19. Rugg-Gunn PJ, Ferguson-Smith AC and Pedersen RA: Epigenetic status of human embryonic stem cells. Nat Genet 37: 585-587, 2005

20. Hikichi T, Kohda T, Wakayama S, Ishino F and Wakayama T: Nuclear transfer alters the DNA methylation status of specific genes in fertilized and parthenogenetically activated mouse embryonic stem cells. Stem Cells 26: 783-788, 2008. 